ORIGINAL STUDY

\title{
Advantages of VMAT-IMRT technique in nasopharyngeal cancer
}

\author{
Cristina Sanda ${ }^{1}$, Codrut Sarafoleanu ${ }^{1,2}$ \\ "Carol Davila" University of Medicine and Pharmacy, Bucharest, Romania \\ ${ }^{2}$ ENT\&HNS Department, "Sfanta Maria” Hospital, Bucharest, Romania
}

\begin{abstract}
BACKGROUND. Radiotherapy, associated with chemotherapy, is the main method of treatment in both early and advanced stages of nasopharyngeal carcinoma. Intensity modulated radiation therapy (IMRT) provides high doses of radiation to the primary tumor, sparing the organs at risk. Volumetric modulated arc therapy (VMAT) is one of the most promising radiation methods which produces superior target coverage, improving the protection of organs at risk and reduces treatment time.

MATERIAL AND METHODS. We performed a retrospective study on 30 patients diagnosed with nasopharyngeal cancer and admitted in "Sfanta Maria" Clinical Hospital between October 2012 and December 2014. All patients have undergone VMATIMRT associated with induction or concurrent chemotherapy. At the end of the treatment, patients were followed up at one, three and six months, and then every six months for 2 years.

RESULTS. At the end of the radio-chemotherapy treatment, 27 patients (90\%) had a complete tumor and lymphatic response and 3 of them $(10 \%)$ presented a partial response. At the end of the follow-up period, we observed 5 patients with recurrences, including 2 deceased.

CONCLUSION. VMAT-IMRT in association with chemotherapy treatment is well tolerated by patients. The good results reflected in high rates of cured patients, the low incidence of side effects, recommend this treatment plan as an optimal indication for nasopharyngeal tumors.
\end{abstract}

KEYWORDS: nasopharyngeal cancer, volumetric modulated arc therapy, intensity modulated radiation therapy

\section{INTRODUCTION}

Nasopharyngeal carcinoma (NPC) is one of the most frequent nasopharyngeal malignant tumors. It is a common pathology among Asians, with a high incidence rate of $20-50$ cases $/ 100,000$ people/year in the Southern part of China, Southeast Asia, North of Africa and Middle East ${ }^{1}$. In non-endemic areas the incidence is below $1 / 100,000$ people/year ${ }^{2}$. The development of NPC seems to be due to the interaction between Epstein-Barr virus (EBV) chronic infection, genetic and environmental factors. In endemic areas, most nasopharyngeal carcinomas are poorly and undifferentiated squamous cell carcinoma, while in western countries the keratinizing type is prevalent ${ }^{3}$.

Being an epithelial tumor, it has a high sensibility to radiotherapy. Therefore, the radiotherapy is the main method of treatment in both early and advanced stages, associated with neoadjuvant and concurrent chemotherapy. Because of its location at the skull base, in close proximity to vital organs (ears, eyes, optic nerve, optic chiasm, temporal lobes, brain stem, salivary glands), multiple radiation techniques were developed to protect those structures. Different studies have shown the advantages and the superior results of intensity modulated radiation therapy (IMRT) over two-dimensional and three-dimensional conformal radiotherapy ${ }^{4-6}$. IMRT can provide high doses of radiation to the primary tumor and decreased doses to the surrounding tissues, reducing the secondary radiation effects and, in this way, improving patients' quality of life.

Volumetric modulated arc therapy (VMAT), implemented by Karl Otto in 2008, is considered to be one of the most promising technologies with a very high potential of rising quality of radiotherapy ${ }^{7}$. It has more flexibility of dose delivery through a full range of angles with continuous modulation of beam aperture and variable dose rate ${ }^{8}$. It produces superior target coverage, improving the protection of organs at risk 
and reduces the treatment time ${ }^{9}$. A hybrid technique combining IMRT and VMAT was developed in order to remove the limits of each method and to improve the dose distribution by increasing the freedom to find the optimal combination of angular sampling and intensity modulation ${ }^{8}$.

\section{MATERIAL AND METHODS}

We analysed 30 patients diagnosed with nasopharyngeal carcinoma in "Sfanta Maria" Clinical Hospital between October 2012 and December 2014. All patients have undergone nasal endoscopic exam and biopsy for histological diagnosis. The patients' evaluation also included a CT scan/MRI of head and neck, chest radiography/CT scan, complete blood count and biochemical profile. All patients were staged according to the TNM system of American Joint Committee on Cancer (AJCC).

All patients have undergone radiotherapy with concurrent chemotherapy +/- induction chemotherapy. In all patients the VMAT-IMRT technique was applied. For CT simulation, a custom head and shoulders mask was used, with the patient in supine position, perform- ing 3-mm-thick CT images from the vertex to $5 \mathrm{~cm}$ inferior to the clavicular heads.

Target volume and normal structures were contoured on axial CT slice using Radiation Therapy Oncology Group (RTOG) guidelines. The CT images were imported into the treatment planning computer (Figure 1).

For radiation therapy planning the Pinnacle ${ }^{3}$ system, version 9.4 , was used, which allows to maximize the target volume prescribed dose and, at the same time, to minimize the critical organs dose. For VMATIMRT an inverse planning was performed (a specific dose was applied for every session of the treatment, according to the total radiation dose prescribed). The obtained results were analysed with the evaluation module of the treatment plan. At this point, a dosevolume histogram is used to show the percentage of the radiation dose received by the volume percent of a structure (Figure 2). It is necessary that the target zone receives enough amount of radiotherapy (at least 95\% of the prescribed dose).

For the critical organs, optimization parameters were established to avoid severe secondary effects that could be triggered. In nasopharyngeal cancer, organs at risk are considered to be the spinal cord, the brainstem, the

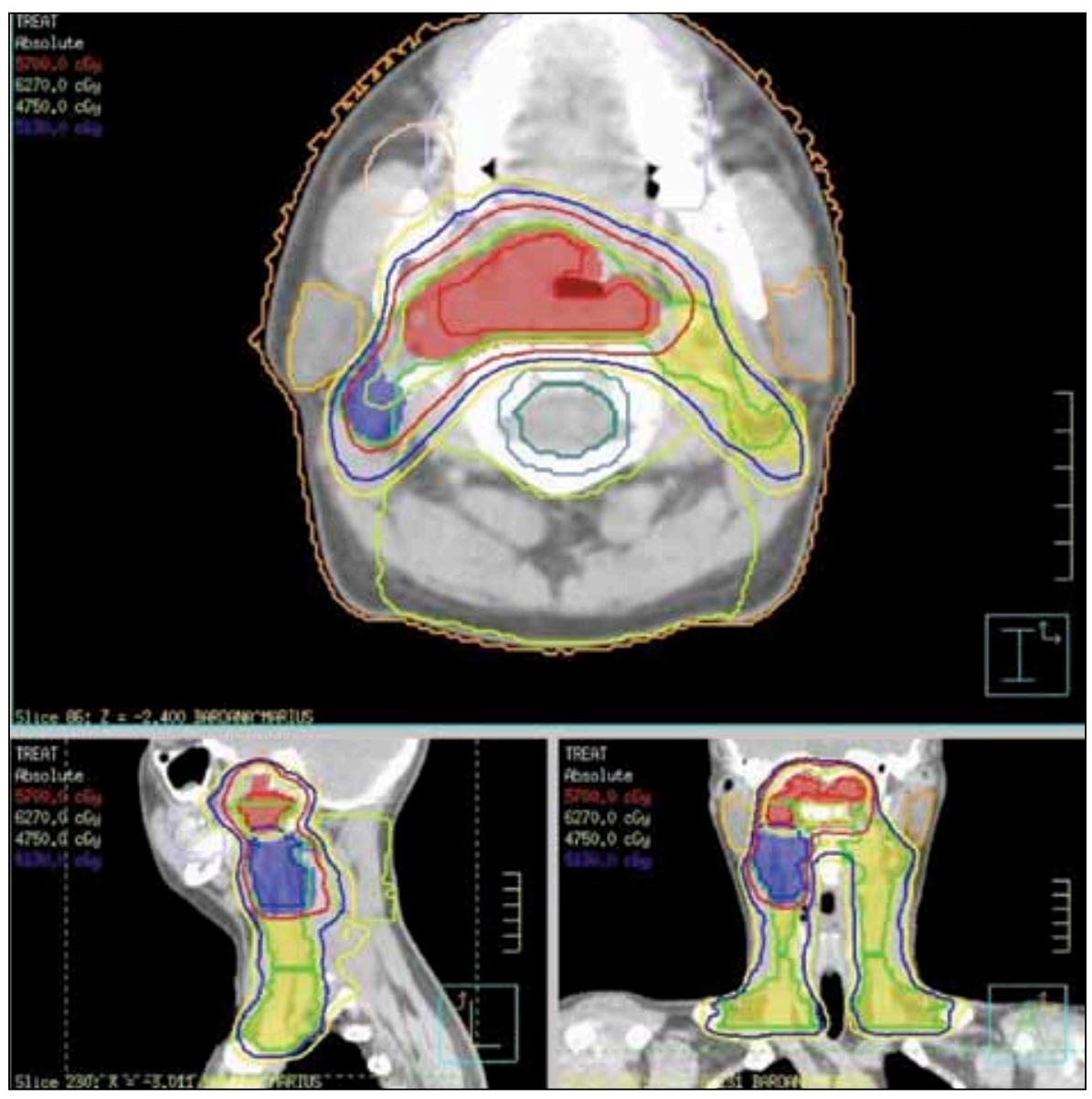

Figure 1 Target volumes and radiation dose distribution of a patient with stage II of the disease 


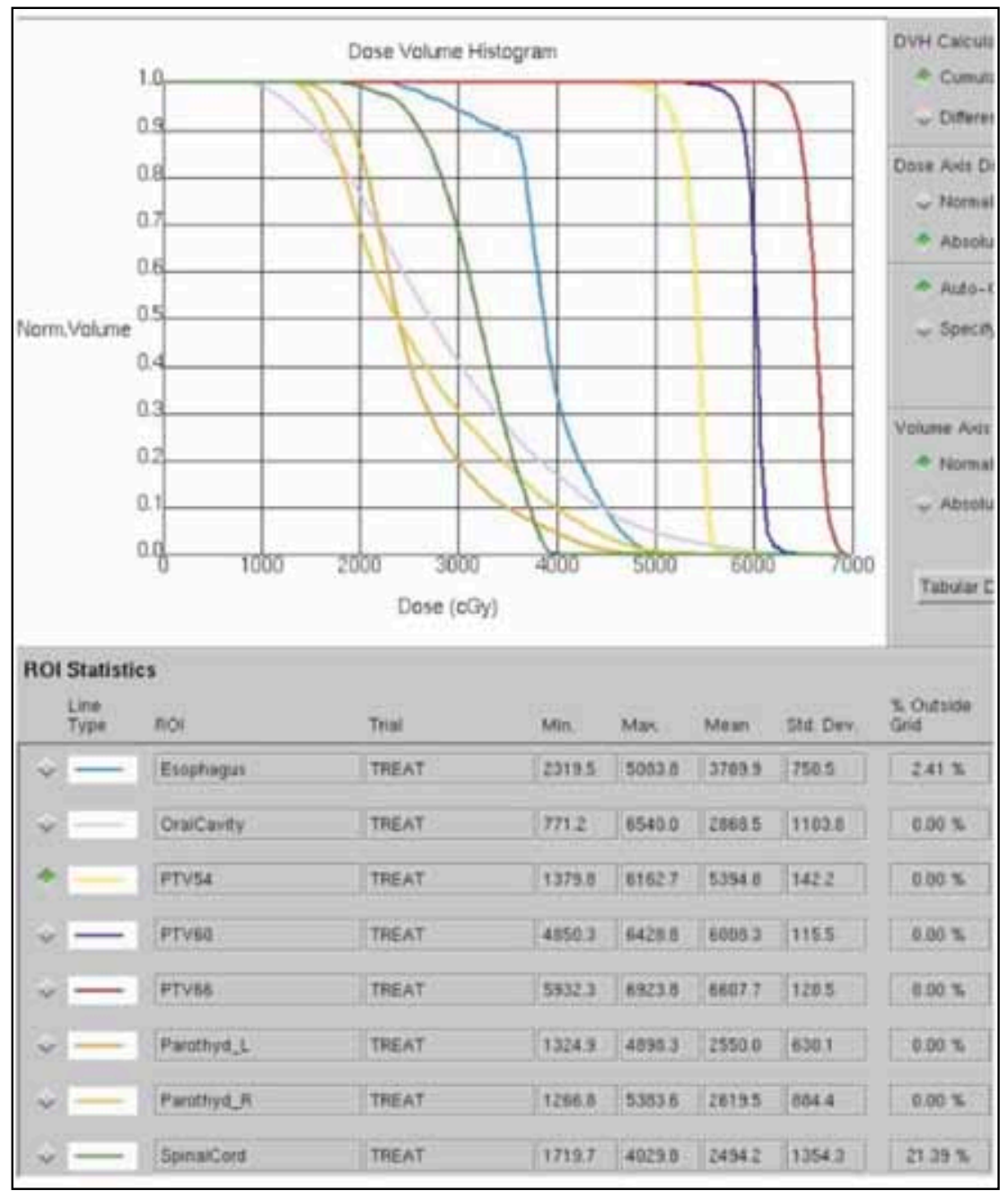

Figure 2 Dose-volume histogram on target volumes and OARs of the same patient with stage II NPC

optic chiasm, the eyes, the optic nerves, the cochlea, the parotid glands, and the maximum doses for them are as follows: $54 \mathrm{~Gy}$ for the brainstem, the optic nerves and the optic chiasm, $45 \mathrm{~Gy}$ for the spinal cord, $<26 \mathrm{~Gy}$ for the parotid glands and $<50 \mathrm{~Gy}$ for the inner ear.

Radiotherapy is a sequential treatment; therefore, the dose prescription was done in two stages:

- I - 25 radiotherapy sessions, a dose of $50 \mathrm{~Gy}$ in daily fractions of 2 Gy was applied to the gross primary tumor and lymphatic system (Planned Target Volume - $\mathrm{PTV}_{50}$ );

- II - 10 radiotherapy sessions, a dose of $20 \mathrm{~Gy}$ in daily fractions of 2 Gy was applied to the primary tumor, resulting in a total dose of $70 \mathrm{~Gy}$ (Planned Target Volume - $\mathrm{PTV}_{70}$ ).

The planned target volume should provide a minimum margin of 3-5 $\mathrm{mm}$ around the clinical target volume $^{1}$.

Induction chemotherapy was administrated in 4-8 series of Docetaxel $50 \mathrm{mg} / \mathrm{m}^{2} /$ day, Cisplatin $100 \mathrm{mg} /$ day or Carboplatin $\mathrm{AUC}=2-4 /$ day and Capecitabine $2000 \mathrm{mg} / \mathrm{m}^{2} /$ day. Chemotherapy administrated con- comitant with radiotherapy included 5-7 series of Cisplatin $40 \mathrm{mg} /$ day or Carboplatin $\mathrm{AUC}=2 /$ day, according to patient's tolerance. In all patients the acute toxicity of the treatment was evaluate.

At the end of the treatment, patients were included in a follow-up control plan, the evaluation being performed at one, three and six months, and then every six months for 2 years. Each visit consisted of clinical and endoscopic examination, complete blood count and biochemical profile, and imaging evaluation (CT scan/MRI) every six months. We evaluated the treatment response at 1, 12, 18 and 24 months after the end of the treatment. In case of persistent disease or tumor recurrence, a biopsy was performed.

\section{RESULTS}

In this study, we analysed 30 patients diagnosed with nasopharyngeal cancer (Figure 3). 22 were men and 8 women, aged between 21-78 years, (median age of 47.5 year, standard deviation $=15.502$ ) . 


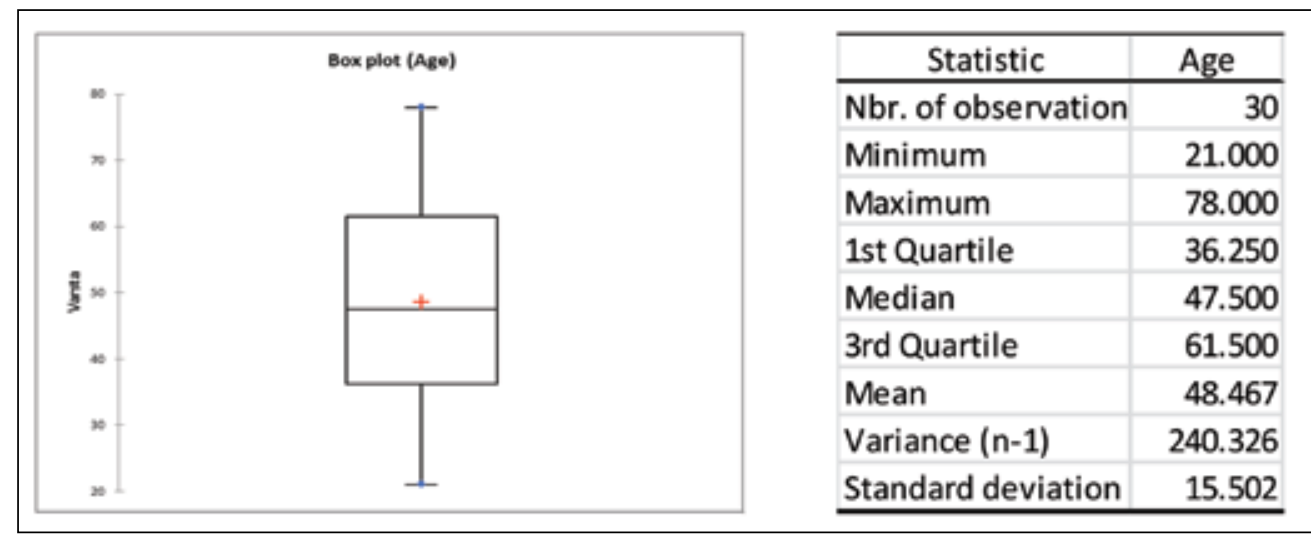

Figure 3 Descriptive statistics of age distribution

All patients were staged according to the TNM system as follows: 2 patients with stage I, 5 patients with stage II, 8 patients with stage III and 15 patients with stage IV (Graphic 1).

According to the World Health Organisation (WHO) classification of nasopharyngeal carcinoma histological type, we found 18 patients with type IIa, 7 patients with type IIb, 1 patient with type I and 4 patients with other histological diagnosis (anaplastic large cell lymphoma, adenoid cystic carcinoma, malignant melanoma, plasmacytic plasmacytoma) (Table 1). Before performing radiotherapy, 19 patients were treated with induction chemotherapy (one patient with stage II, 6 patients with stage III and 12 patients with stage IV), while 27 patients underwent concurrent chemoradiotherapy and 3 patients only radiotherapy.

At the end of the radio-chemotherapy treatment, 27 patients $(90 \%)$ showed complete tumor and lymphatic

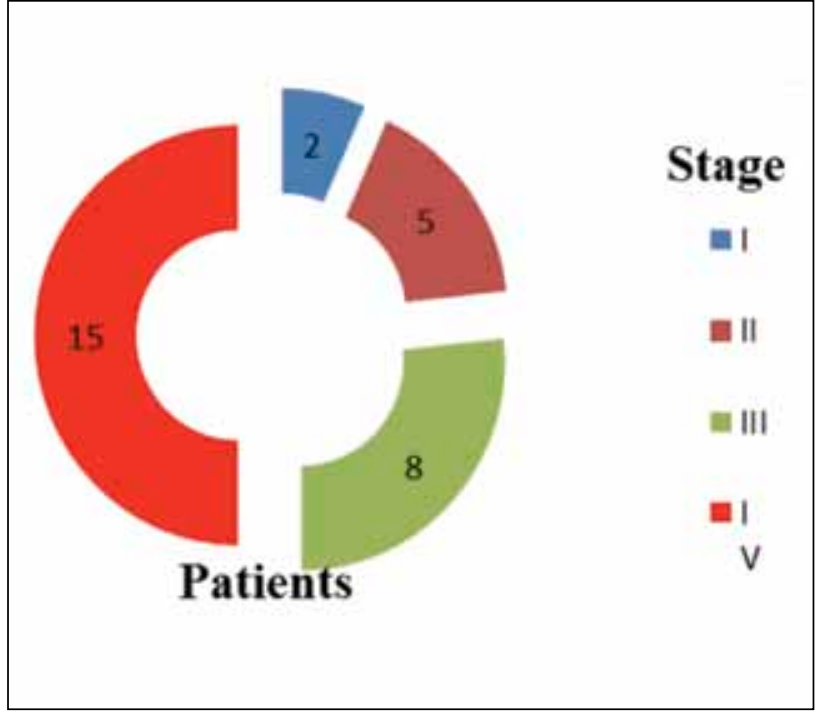

Graphic 1 Patients' distribution regarding TNM stage

\section{Table 1}

\section{Patients' characteristics}

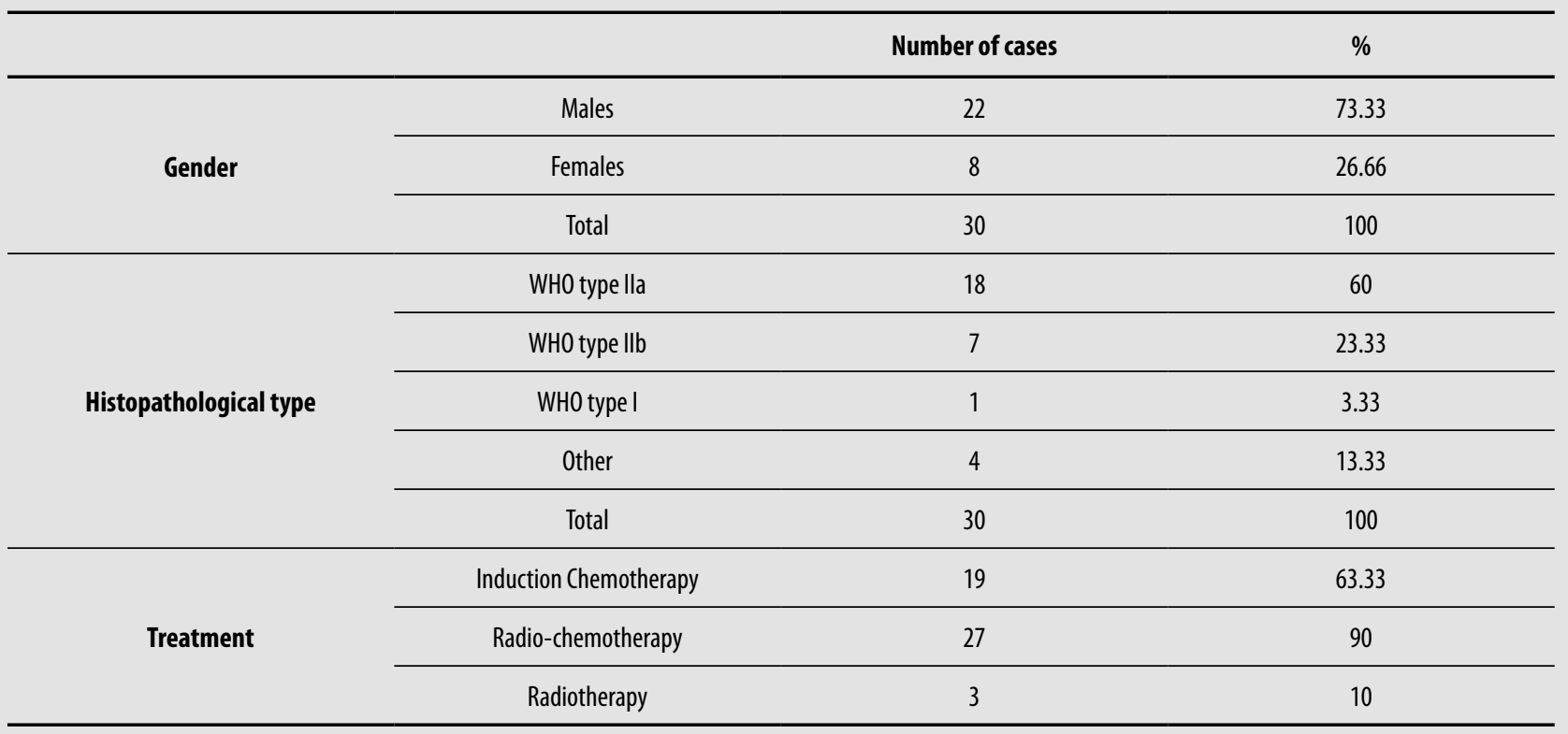


response and 3 of them (10\%) presented a partial response: a 21-year-old male with stage III of disease $\left(\mathrm{cT}_{2} \mathrm{~N}_{2} \mathrm{M}_{0}\right)$ had complete tumor response, but progressive disease at cervical nodes; a 73-year-old male with stage IVA of disease $\left(\mathrm{cT}_{4 \mathrm{~b}} \mathrm{~N}_{2 \mathrm{~b}} \mathrm{M}_{0}\right)$ presented a reduction of the tumor mass and complete lymphatic response; a 48-year-old female with stage IVA $\left(\mathrm{cT}_{4} \mathrm{~N}_{2} \mathrm{M}_{0}\right)$ showed important decrease of the tumor volume.

We evaluated the treatment response at 1, 12, 18 and 24 months after the end of the treatment. It was observed that 25 patients were tumor-free at 24 months, 2 deceased and 3 with recurrences (Table 2).

Although at the end of the treatment 27 patients $(90 \%)$ were free of tumor, at the end of the follow-up period $25(83.33 \%)$ patients had complete response.

All patients completed the radio- and chemotherapy without any interruption. Those patients with high tox- icity and bad tolerance of the treatment received supportive nutritional therapy in order to avoid discontinuity of the oncologic treatment. In Table 3 we analysed the acute complication of chemo-radiotherapy, depending on the toxicity grade and patient stage.

Our study also shows decreasing rates of acute toxicity during the radiotherapy. Only 4 patients had grade III dermatitis, 4 patients presented grade III xerostomia. Grade III of mucositis or dysphagia was observed in 2 , respectively 4 patients.

It is important to mention that adverse effects of radio-chemotherapy like dermatitis, mucositis and xerostomia were correlated with TNM degree (significance level $\mathrm{p}$-values $<0.05)$. In our study, dysphagia did not correlate with TNM degree ( $p$-value $>0.05$ ) but was well correlated with mucositis $(p=0.02)$ (Table 4).

Table 2

Treatment response in the follow-up period

\begin{tabular}{cccc}
\hline Evaluation & Tumor-free & Recurrence & Deceased \\
\hline 1st month & 27 & 3 & 0 \\
\hline 12 months & 26 & 4 & 0 \\
\hline 18 months & 25 & 5 & 0 \\
\hline 24 months & 25 & 3 & 2 \\
\hline
\end{tabular}

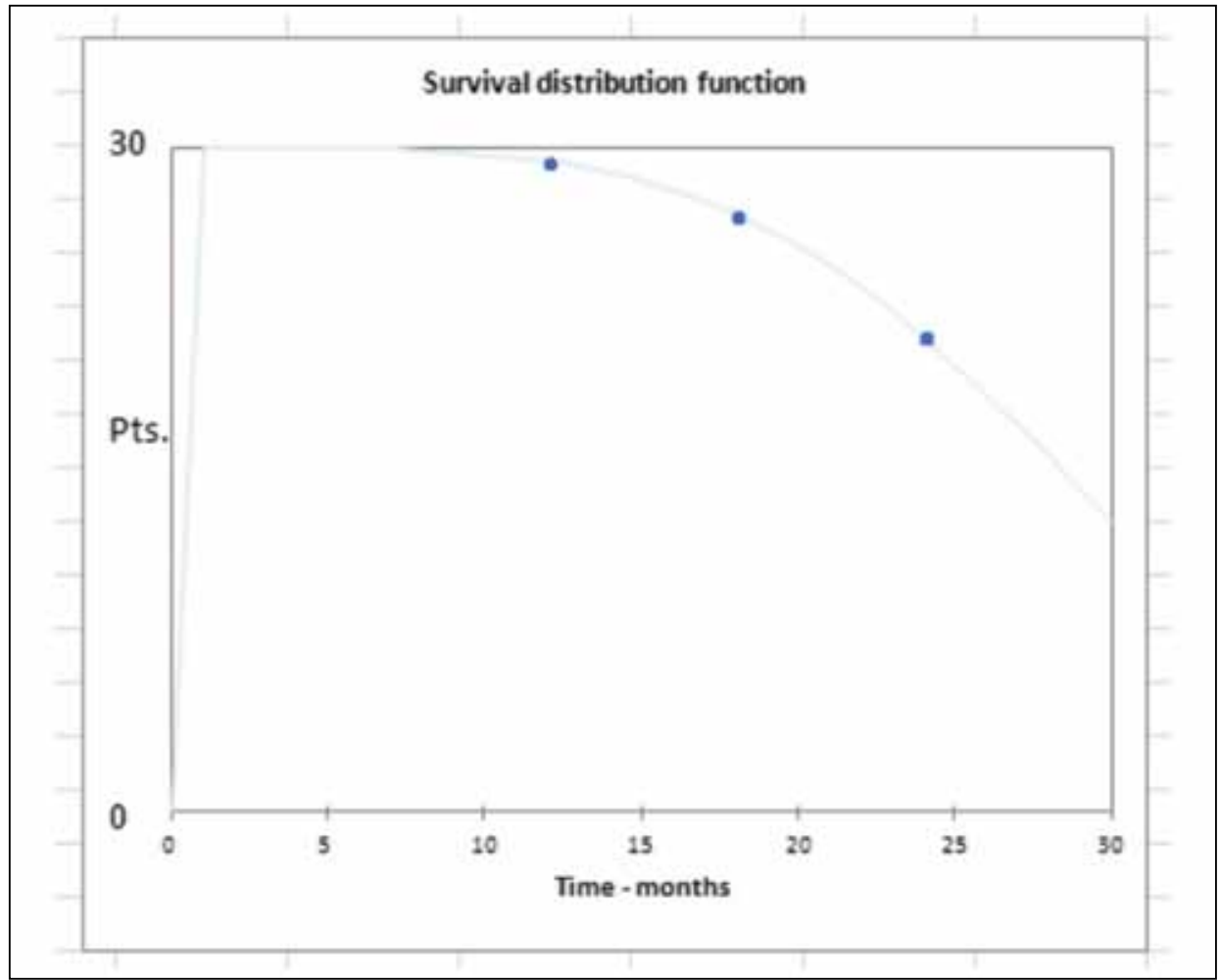

Graphic 2 Complete treatment response during the follow-up period 
Table 3

The acute complication of chemo-radiotherapy, depending on the toxicity grade and patient stage

\begin{tabular}{|c|c|c|c|c|c|c|}
\hline \multicolumn{7}{|c|}{ VMAT-IMRT } \\
\hline \multicolumn{2}{|c|}{ TNM stage } & I & II & III & IV & Total patients \\
\hline \multirow{5}{*}{ Dermatitis } & Grade I & 1 & - & 1 & 6 & 8 \\
\hline & Grade II & 1 & 4 & 4 & 1 & 10 \\
\hline & Grade III & - & 1 & 1 & 2 & 4 \\
\hline & Grade II & - & 2 & 3 & 1 & 6 \\
\hline & Grade III & - & - & - & 2 & 2 \\
\hline \multirow{3}{*}{ Xerostomia } & Grade I & 2 & 3 & 3 & 4 & 12 \\
\hline & Grade II & - & 2 & 2 & 5 & 9 \\
\hline & Grade III & - & - & 1 & 3 & 4 \\
\hline \multirow{3}{*}{ Dysphagia } & Grade I & 2 & 2 & 1 & 4 & 9 \\
\hline & Grade II & - & 1 & 4 & 1 & 6 \\
\hline & Grade III & - & 2 & - & 2 & 4 \\
\hline
\end{tabular}

Table 4

Table of correlation between adverse effects of radio-chemotherapy

\begin{tabular}{|c|c|c|c|c|c|}
\hline Variables & TNM & Dermatita & Mucositis & Xerostomia & Dysphagia \\
\hline TNM & 0 & 0.006 & 0.016 & 0.046 & 0.061 \\
\hline Dermatitis & 0.006 & 0 & 0.014 & 0.022 & 0.066 \\
\hline Mucositis & 0.016 & 0.014 & 0 & 0.022 & 0.020 \\
\hline Xerostomia & 0.046 & 0.022 & 0.022 & 0 & 0.065 \\
\hline Dysphagia & 0.061 & 0.066 & 0.020 & 0.065 & 0 \\
\hline
\end{tabular}

Values in bold are different from 0 with a significance level alpha $=0.05$

\section{DISCUSSIONS}

The treatment of nasopharyngeal cancer consists mainly in radiotherapy associated with neoadjuvant and concurrent chemotherapy.
The necessity of using hybrid VMAT-IMRT technique in nasopharyngeal cancer emerged from the need to reduce the weak points of each technique. In IMRT the conformity of dose distribution is often limited because of the dissipated angular sampling, 
while VMAT has sufficient angular sampling; it did not provide the desired intensity modulation in some beam directions. Therefore, the hybrid VMAT-IMRT technique improve the dose distribution by finding the best combination of angular sampling and intensity modulation and, in this way, allows the administration of a high dose of radiation to the primary tumor, providing, at the same time, an adequate protection of the critical structures. There are studies which demonstrated the efficacy of association of these two techniques ${ }^{8,10}$.

It was demonstrated that $\mathrm{PTV}_{70}$ applied by another radiation technique, as $3 \mathrm{D}$ conformal radiotherapy, may produce severe side effects, like radiation myelitis ${ }^{11,12}$.

Also, VMAT-IMRT presents a better conformity index and a homogeneity index for both $\mathrm{PTV}_{70}$ and $\mathrm{PTV}_{50}$, compared with other radiation techniques ${ }^{8,13}$.

In the current study, VMAT - IMRT was used in every patient. At the end of the treatment, $90 \%$ of patients had complete response, and only $10 \%$ of patients achieved a partial response.

The association of radiotherapy with chemotherapy can improve the loco-regional control rate ${ }^{14}$, but it may also increase the toxicity (hematologic toxicity, oral mucositis). The capacity of IMRT in reducing doses of irradiation in non-target tissues decreases also the rates of producing acute toxicity. Multiple studies indicate a lower incidence of acute toxicity of radiotherapy $y^{15,16}$.

For those patients with low tolerance of the treatment, supportive nutrition can be administered, in order to prevent therapy interruption.

In our study, the disease response, confirmed by clinical and imagistic examination in the follow-up period, were comparable with other studies involving IMRT or VMAT-IMRT ${ }^{17,18}$.

There are some limitations of this study. The implementation of VMAT-IMRT in Romania for about 3 years determined a short follow-up for our analysis. We should know the survival rate at 5 years after the treatment. To assess the role of VMAT-IMRT in the locoregional control of nasopharyngeal cancer further prospective studies with bigger sample size are needed.

\section{CONCLUSIONS}

VMAT-IMRT in association with chemotherapy treatment is well tolerated by patients. The good results reflected in high rates of cured patients, the low incidence of side effects, recommend this treatment plan as an optimal indication for nasopharyngeal tumors.

Conflict of interest: The authors have no conflict of interest.

Contribution of authors: All authors have equally contributed to this work.

\section{REFERENCES}

1. Ma j., Cao S. - The epidemiology of nasopharyngeal carcinoma. In: Lu J.J., Cooper J.S., Lee A.W.M. (Eds.) - Nasopharyngeal Cancer: Multidisciplinary Management. Springer-Verlag Berlin Heidelberg, 2010;p.1-8.

2. Parkin D.M., Whelan S.L., Ferlay J., Raymond L., Young J., editors. Cancer incidence in five continents. Volume VII. IARC, 1997;Sci Publ no. 143 .

3. Marks J.E., Phillips J.L., Menck H.R. - The National Cancer Data Base report on the relationship of race and national origin to the histology of nasopharyngeal carcinoma. Cancer, 1998;83(3):582-588.

4. Cheng J.C., Chao K.S., Low D. - Comparison of intensity modulated radiation therapy (IMRT) treatment techniques for nasopharyngeal carcinoma. Int J Cancer, 2001;96(2):126-131.

5. Kam M.K., Chau R.M., Suen J., Choi P.H., Teo P.M. - Intensity-modulated radiotherapy in nasopharyngeal carcinoma: Dosimetric advantage over conventional plans and feasibility of dose escalation. Int J Radiat Onc Biol Phys., 2003;56(1):145-157.

6. Xia P., Fu K.K., Wong G.W., Akazawa C., Verhey L.J. - Comparison of treatment plans involving intensity-modulated radiotherapy for nasopharyngeal carcinoma. Int J Radiat Oncol Biol Phys., 2000;48(2):329337.

7. Otto K. - Volumetric modulated arc therapy: IMRT in a single gantry arc. Med Phys., 2008;35(1):310-317.

8. Zhao N., Yang R., Jiang Y., Tian S., Guo F., Wang J. - A Hybrid IMRT/ VMAT Technique for the Treatment of Nasopharyngeal Cancer. BioMed Research International, 2015;(2015). Article ID 940102.

9. Verbakel W.F., Cuijpers J.P., Hoffmans D., Bieker M., Slotman B.J., Senan S. - Volumetric intensity-modulated arc therapy vs. conventional IMRT in head-and-neck cancer: a Comparative Planning and Dosimetric Study. Int J Radiat Oncol Biol Phys., 2009;74(1):252-259. doi: 10.1016/j. ijrobp.2008.12.033.

10. Zhao N., Yang R., Wang J. - A Novel Integrated VMAT/IMRT Technique for the Treatment of Nasopharyngeal Carcinoma. Int J Radiat Oncol Biol Phys., 2014;90(1 Suppl):S937-S938.

11. Kwong D., McMillan A., Pow E., Sham J. - A randomized trial comparing intensity modulated radiotherapy versus 2-dimensional radiotherapy for stage II nasopharyngeal carcinoma. Int J Radiat Oncol Biol Phys., 2008;72(1 Suppl):S98.

12. Lee A.W., Law S.C., Ng S.H., Chan D.K., Poon Y.F., Foo W., Tung S.Y., Cheung F.K., Ho J.H. - Retrospective analysis of nasopharyngeal carcinoma treated during 1976-1985: late complications following megavoltage irradiation. Br J Radiol., 1992;65(778):918-928.

13. Kristensen C.A., Kjær-Kristoffersen F., Sapru W., Berthelsen A.K., Loft A., Specht L. - Nasopharyngeal carcinoma. Treatment planning with IMRT and 3D conformal radiotherapy. Acta Oncol., 2007;46(2):214220.

14. Langendijk J.A., Leemans C.R., Buter J., Berghof J., Slotman B.J. - The additional value of chemotherapy to radiotherapy in locally advanced nasopharyngeal carcinoma: A meta-analysis of the published literature. J Clin Oncol., 2004;22(22):4604-4612.

15. Lee N., Xia P., Quivey J.M., Sultanem K., Poon I., Akazawa C., Akazawa P., Weinberg V., Fu K.K. - Intensity-modulated radiotherapy in the treatment of nasopharyngeal carcinoma: an update of the UCSF experience. Int J Radiat Oncol Biol Phys., 2002;53(1):12-22.

16. Kim J.W., Cho J.H., Keum K.C., Kim J.H., Kim G.E., Lee J.Y., Kim S.K., Lee C.G. - IMRT with Simultaneous Integrated Boost and Concurrent 
Chemotherapy for Nasopharyngeal Cancer: Plan Evaluation and Treatment Outcome. Jpn J Clin Oncol., 2012;42(12):1152-1160. doi: 10.1093/jjco/hys169. Epub 2012 Oct 16.

17. Guo R., Tang L.L., Mao Y.P., Zhou G.Q., Qi Z.Y., Liu L.Z., Lin A.H., Liu M.Z., Ma J., Sun Y. - Clinical Outcomes of Volume-Modulated Arc Therapy in 205 Patients with Nasopharyngeal Carcinoma: An Analysis of Survival and Treatment Toxicities. PLoS One, 2015;10(7):e0129679. doi: 10.1371/journal.pone.0129679. eCollection 2015.

18. Saleh-Ebrahimi L., Zwicker F., Muenter., Marc W., Bischof M., Lindel K., Debus J., Huber P.E., Roeder F. - Intensity modulated radiotherapy (IMRT) combined with concurrent but not adjuvant chemotherapy in primary nasopharyngeal cancer - a retrospective single center analysis. Radiation Oncology, 2013;8:20. doi: 10.1186/1748-717x-8-20. 